\title{
Tuberculosis Occupational Risks in Field-based Health Care Workers in India
}

\author{
Senbagavalli Prakash Babu, Sonali Sarkar*
}

\section{Senbagavalli Prakash Babu, Sonali Sarkar*}

Department of Preventive and Social Medicine, JIPMER, Pondicherry, INDIA.

Correspondence

Prof. Sonali Sarkar

Additional Professor, Preventive and

Social Medicine, JIPMER, Gorimedu,

Puducherry, INDIA.

Phone no: +91-9442174663

Email: sarkarsonaligh@gmail.com

DOI : 10.5530/ijmedph.2019.2.9

Article Available online

http://www.ijmedph.org/v9/i2

Copyright

(C) 2019 Phcog.Net. This is an openaccess article distributed under the terms of the Creative Commons Attribution 4.0 International license.
Health care workers are the most valuable resource in biomedical research and the World Health Organization (WHO) had declared the years 2006 to 2015 as the "The decade of the human resources for health". The target of tuberculosis (TB) elimination globally is 2030, but India has proposed to eliminate it by 2025 . TB research and development is essential to achieve the global TB targets set in the Sustainable Development Goals and the End TB Strategy. One of the pillars proposed by WHO for END TB strategy is intensified research and innovation. ${ }^{1}$ Priorities for TB research and development include vaccines to lower the risk of infection, a vaccine or new drug treatment to reduce the risk of TB disease in already latently infected, rapid diagnostics for use at the point of care and simpler and shorter drug regimens for treating TB disease. India is working hard towards intensifying research in TB with a recent surge in field-based research for the discovery and development of newer tools and their implementation; interventions and to develop strategies. Treatment for TB is domiciliary and community-based interventions are mandatory for the prevention and care. Field-based health workers (FHW) including Medical Social Workers, Nurses, RNTCP workers and DOTS providers play key role in the treatment for tuberculosis, enhancing active case finding and intensifying research and innovation. The risk that Mycobacterium tuberculosis can be transmitted from patients with active tuberculosis (TB) to other patients and healthcare workers has been recognized for many years. ${ }^{2}$ It is high-time that proper safety measures are framed to prevent and support FHW working in tuberculosis. The level of risk varies by setting, occupation, patient population and effectiveness of TB infection control measures. If average community based annual risk of infection in India is about $1.5 \%$, healthcare workers have additional $3.5 \%$ risk, which may be attributable to nosocomial exposure. ${ }^{3}$

Direct health hazards in field range from respiratory aerosols from patients during consenting and induction of cough and sputum to needle pricks during blood draw. In a report published by the WHO, the disease burden caused by percutaneous sharps injuries among healthcare workers was found to be three million per year. Despite this, almost $80 \%$ of healthcare workers remain unimmunized (against Hepatitis B) in many parts of the world. ${ }^{4}$ Other minor hazards include exposure to long travelling hours, noise and ergonomic issues such as heavy lifting of field-bags and standing for long periods. The fear of contracting infectious diseases and or transmitting to their family might lead to development of stress in FHW.

Tests used to detect latent infection including tuberculin skin test and Interferon-gamma release assays have lot of diagnostic significance in identifying latent $\mathrm{TB}$ infection but also come with certain drawbacks. Treatment of LTBI is not well studied in these specific risk groups. Several action points come to mind. Effective environmental and personal protective measures along with education to the patients and the HCW needs to be carried out expeditiously. Immunization status especially to Hepatitis $B$ should be updated and appropriate immunization should be provided to the field personnel. Good ventilation without recirculation of air is essential to reduce the risk of aerosols and unfortunately majority of the under-resourced community health care facilities lack such a set up to be rendered to field staff during sample collection. Long-term research projects should tailor budget for the health welfare of the FHW involved in the study.

Proper biomedical waste management is essential especially in field-based research projects. It is mandatory to train and assess the knowledge level of biomedical waste management of the FHW. Frequent behavioural assessments and proper counselling should be given to all HCW. Safety controls also includes strict administrative policies for the safety of field employees, regular TB screening, appropriate support, possible employment security and compensation for HCWs with tuberculosis. Environmental measures such as adequate rooms for consenting and blood draw from patients with natural or artificial ventilators should be enabled. Staff should be fit-tested for N-95 respirators and supply of respirators should be ensured to the employee with no re-use of the ventilators and proper discarding after use.

The ability of the FHW to follow the TB prevention guidelines and its impact on their attitudes towards TB patients should be critically assessed. Health care workers are key stakeholders for interventions to prevent occupationally acquired TB.

The population served by the health care facility and the tuberculous infection risk in the community 
where the health care workers (HCW) reside are strong variables influencing the amount of potential tuberculosis exposure of the employees. The tuberculin test conversion rate in hospital-based set up was $3.2 \%$, whereas employees working in non-contact jobs had a rate of $7.8 \%{ }^{5}$ Therefore, it is likely that nonhospital sources of infection do play a considerable role in the acquisition of infection. The available literature does not provide much information in comparing the workplace risk versus the community in which HCW reside.

There is a paucity of research into the hazards faced by healthcare workers in our country and we need to expand the scope of this research from single centre studies to the analysis of data from multiple centres. This alone will provoke interventions that are designed for large scale implementation. The need of the hour is to prioritize occupational health of community-based health workers and ensure that the workforce is adequately trained and stay healthy. As humanitarians and experts of public health, it is our duty to concentrate and help disease risk groups.

\section{REFERENCES}

1. WHO, Global Tuberculosis Report. 2018.

2. Sepkowitz KA. Tuberculosis and the health care worker: a historical perspective. Ann Intern Med. 1994;120(1):71-9.

3. Aggarwal AN. Tuberculosis transmission at healthcare facilities in India. Lung India. 2009;26(2):33-4. doi: 10.4103/0970-2113.48893.

4. Joseph B, Joseph M. The health of the healthcare workers. Indian J Occup Environ Med. 2016;20(2):71-2. doi: 10.4103/0019-5278.197518.

5. Daniel TM. The Occupational Tuberculosis Risk of Health Care Workers. Tuberculosis in the Workplace. National Academies Press. 2001. 